\title{
Politique publique et action collective territoriale. Une analyse de la politique environnement-emploi de la Région Nord-Pas de Calais
}

\section{Muriel Maillefert et Nicola Screnci}

\section{(2) OpenEdition}

Édition électronique

URL : http://journals.openedition.org/developpementdurable/7963

DOI : 10.4000/developpementdurable.7963

ISSN : 1772-9971

Éditeur

Association DD\&T

\section{Référence électronique}

Muriel Maillefert et Nicola Screnci, « Politique publique et action collective territoriale. Une analyse de la politique environnement-emploi de la Région Nord-Pas de Calais », Développement durable et territoires [En ligne], Varia (2004-2010), mis en ligne le 30 janvier 2009, consulté le 01 mai 2019. URL: http://journals.openedition.org/developpementdurable/7963 ; DOI : 10.4000/ developpementdurable.7963

Ce document a été généré automatiquement le 1 mai 2019.

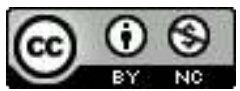

Développement Durable et Territoires est mis à disposition selon les termes de la licence Creative Commons Attribution - Pas d'Utilisation Commerciale 4.0 International. 


\title{
Politique publique et action collective territoriale. Une analyse de la politique environnement- emploi de la Région Nord-Pas de Calais
}

\author{
Muriel Maillefert et Nicola Screnci
}

1 Depuis une vingtaine d'années, le domaine de la politique publique s'est segmenté et fractionné. Aux grands modèles macro-économiques très globaux se sont substituées des analyses et évaluations souvent très spécifiques ou très spécialisées pouvant parfois déboucher sur des recommandations de politique économique très fortes ${ }^{1}$. Dans le domaine de la politique de l'emploi, les évolutions les plus sensibles se sont exprimées en termes de territorialisation, la compétence générale restant dévolue à l'Etat (Berthet et al. , 2004). De ce fait, le point de vue de l'Etat central est resté prédominant et les analyses ont le plus souvent et assez logiquement adopté ce même point de vue (Maillefert, 2002a).

Malgré des résultats riches en matière d'évaluation des politiques publiques, et l'acquisition d'information sur la question de la territorialisation, une telle démarche ne permet pas, par sa nature même, d'expliciter de manière satisfaisante la dimension territoriale : la variable locale est ramenée à un simple comportement d'acteurs localisés, sans que la variable « territoire » n'acquière de dimension explicative propre. Analyser la territorialisation se ramène alors à l'analyse des processus verticaux de déconcentration des politiques à l'échelon des territoires ou à l'analyse des interactions des individus sur un territoire donné (Maillefert, 2002b).

3 Au-delà de l'analyse des politiques publiques, certains travaux d'économie régionale (notamment ceux liés au courant de la proximité) tentent de donner consistance à l'idée de territoire, le territoire étant un construit à la conjonction des dimensions géographiques, économiques et sociales. Mais ces derniers ne sont pas spécialisés sur l'analyse des politiques publiques, et considèrent l'emploi ou les politiques du marché du 
travail essentiellement du point de vue des systèmes productifs (Pecqueur et Zimmerman, 2004). L'effort d'analyse se concentre alors sur l'émergence et la durabilité des «rencontres productives", les politiques publiques étant seulement appréhendées comme une composante de l'analyse.

L'analyse des politiques publiques et des politiques d'emploi a beaucoup évolué depuis vingt-cinq ans. Un résultat semble acquis : il est temps de ne plus considérer l'action publique sous un angle exclusivement sectoriel (analyse d'une politique publique particulière), ou hiérarchique (analyse de la décentralisation ou de la déconcentration comme une opposition entre le global/et le local). C'est vrai pour de nombreuses politiques publiques pour plusieurs raisons. D'une part, l'action publique s'est largement territorialisée sous l'influence des différentes législations intervenues depuis le début des années quatre-vingt. D'autre part, les politiques publiques à l'échelle territoriale telles qu'on les observe ne sont pas nécessairement spécialisées parce que les collectivités qui les prennent en charge ne peuvent ou ne veulent pas se restreindre à ce type de démarche. Le plus souvent, en effet, elles ne disposent pas de la compétence en question, alors même qu'on en attend des résultats sur un terrain précis (c'est le cas précisément des questions de politique d'emploi). C'est pourquoi des croisements thématiques sont de plus en plus souvent observés à l'échelon local. Un de ces croisements concerne les politiques associant développement de l'emploi et protection de l'environnement, politiques souvent appelées politiques emploi-environnement.

Un des acteurs principaux de cette déspécialisation semble être la Région, qui, par bien des aspects joue un rôle d'acteur-clé (au sens de la théorie de la proximité) dans cette évolution. En effet, aujourd'hui en France, les collectivités territoriales, et notamment l'institution régionale, assument un rôle majeur aussi bien dans la réalisation des politiques environnementales que dans les politiques/actions sociales à l'échelle locale, mais leur implication dans les projets prend une figure spécifique à chaque fois.

6 L'objectif de cette contribution est de proposer une réflexion sur la question de l'interconnexion entre les politiques d'emploi et d'environnement dans un cadre territorial (avec comme terrain particulier la Région Nord-Pas-de Calais). Elle se fonde sur une analyse des politiques régionales emploi-environnement menées entre 1993 et 2002 en Région, dans le but de contribuer à expliquer les logiques d'action collective des différents acteurs en présence et le rôle de la variable territoriale. Après avoir présenté les grandes lignes du dispositif emploi-environnement, nous essayons, à l'aide d'une méthodologie spécifique, de décrypter les ressorts de l'action collective territoriale. En particulier, nous montrons que les acteurs publics régionaux et territoriaux ont développé des registres spécifiques d'action collective, qui expliquent en grande partie l'échec de la politique et son recentrage final. Toutefois l'expérience de cette politique publique a permis de construire et stabiliser, par ce biais même, certains éléments constitutifs d'une politique de développement territorial en aidant à l'émergence d'objectifs régionaux qui sont aujourd'hui beaucoup plus explicites.

1. La politique régionale emploi-environnement du NPC

7 Initiée en 1993, la politique emploi-environnement de la Région Nord-Pas de Calais (Région NPC) s'est appuyée sur le double constat d'une situation environnementale très dégradée, et de l'existence d'un volant de chômage élevé. Suite à l'arrivée aux affaires d'une équipe politique verte, un pari a été engagé de promouvoir la création d'emplois répondant au double défi du chômage et de la reconquête environnementale. Entre 1993 et 2002, trois vagues de dispositifs ont été expérimentés, soit en collaboration avec les 
politiques de l'Etat, soit en complément de ces politiques. La lecture des dispositifs montre une évolution progressive, mais irréversible vers un recentrage autour des politiques plus traditionnelles de soutien à l'activité économique. Elle s'est opérée après une hésitation entre l'orientation autour de l'entrée emploi, puis de l'entrée environnement.

\subsection{Une expérimentation originale}

arrage de l'implication territoriale dans la politique d'emploi peut être rattaché au programme Contrats Verts mis en place par la loi du 27 juillet 1993 relative au développement de l'emploi de l'apprentissage. En effet, il était prévu qu'à titre expérimental, les Conseils Régionaux mènent des actions créatrices d'emplois dans le domaine de l'environnement, l'objectif étant de créer à l'échelon national, 35000 emplois verts sur 2 ans $^{2}$. Une campagne de promotion Etat - Région s'est déroulée en 1994, l'objectif était clairement d'entreprendre une expérience, limitée dans le temps sur deux ans et pas forcément renouvelable, d'aide à la création d'emploi. L'instauration d'un partenariat actif Etat / Région a essentiellement émergé en phase de démarrage du programme. Certaines Régions ont parfois souhaité définir leurs propres critères d'intervention, et, prenant le relais de l'Etat, ont ainsi mobilisé des crédits sur leurs propres budgets et intégré les « emplois verts » dans leur politique régionale.

Le Conseil Régional Nord-Pas de Calais en concertation avec la DIREN a lancé en 1994, un programme Emploi-Environnement, avec comme objectif la création d'emplois par émergence d'actions de protection, de requalification, d'entretien de l'environnement et du cadre de vie (cf. plaquette publiée en 1994 et diffusée en octobre 1994 auprès des associations d'environnement, des collectivités, des administrations, de l'ANPE et des Missions Locales).

L'opération «emplois verts » de l'Etat s'est poursuivie en 1995 sous forme d'une aide de l'Etat à des emplois d'insertion à destination des collectivités locales et des associations dans le domaine de l'environnement, alors même que la Région Nord - pas de Calais, invoquant divers arguments (rythme jugé trop rapide d'engagement des crédits d'État, risques d'un certain saupoudrage des crédits, difficulté à ne pas se contenter d'accompagner des projets avec des Contrats Emploi Solidarité, volonté de construire des projets tendant à créer de l'emploi pérenne) choisissait de mener une politique distincte de celle de l'Etat. Le programme régional est infléchi en 1996, en direction du secteur environnemental et de la création d'emploi associée.

11 On perçoit donc, dès les premières années du dispositif, une divergence entre l'action publique territorialisée (matérialisée par les emplois verts de l'Etat) et l'action publique régionale. Cette divergence, qui s'exprime par une démarcation entre action régionale et action publique décentralisée est justifiée, du point de vue de l'acteur régional, par deux arguments: une volonté de se démarquer de la logique d'insertion, d'une part, et de se concentrer sur la création d'activité dans le domaine de l'environnement, de l'autre.

12 L'évolution de la politique Régionale se traduit à cette époque par une modification des critères de sélection des projets aidés. Ces derniers sont soumis à un critère de ciblage plus strict, alors même que les aides sont recentrées. Les aides deviennent plus exigeantes autant du point de vue des critères concernant l'emploi, que ceux concernant l'environnement. Ainsi des critères comme «la qualité environnementale et sociale du champ d'action» et "la qualité des formations et des compétences mobilisées» apparaissent explicitement, alors même que la mesure emblématique du nouveau 
programme, le soutien à la création d'un corps d'écogardes dans les parcs naturels régionaux, fait l'objet d'une opération de communication à grande échelle.

La réorientation de la politique se poursuit après 1997, d'autant que, suite au rapport Hascoet (1997), le programme Nouveaux Services-Emplois Jeunes (NS-EJ) est mis en place, avec un ciblage assez proche de la politique régionale, notamment en termes de domaine (mais moins en termes de public). Une dernière évaluation menée au début des années 2000 sonne finalement le glas de la politique spécifique emploi-environnement, l'action de la Région se réorientant définitivement vers des mesures plus traditionnelles de soutien à l'activité économique.

La justification de cette réorientation apparaît clairement dans les documents du Conseil Régional. Bien que les acteurs régionaux continuent à appuyer l'orientation en faveur de ce qu'ils nomment «l'excellence environnementale», l'environnement n'est plus considéré comme un secteur cible de mesures spécifiquement financées par la Région, qui considère que le coût des mesures ne justifie plus un investissement financier jugé trop lourd. Par contre, l'environnement n'est pas abandonné, mais est analysé comme faisant partie d'une thématique transversale devant être intégrée dans toutes les actions de développement économique. D'une certaine façon, cette inflexion de la politique régionale traduit le fait que la Région abandonne la politique environnementale sectorielle au profit d'une politique environnementale transversale, celle-ci étant résumée par une référence générique au développement durable. Cette réorientation radicale de la politique régionale se fonde sur un constat d'échec lié à deux évaluations successives du dispositif. L'étude de l'argumentaire ayant justifié cette réorientation constitue un matériau riche pour l'interprétation de la politique publique régionale.

1.2 Des résultats mitigés

15 La politique emploi-environnement a été évaluée par deux fois : en 1997 et en 2002. La première évaluation a porté sur les années 1995 et 1996, alors que la seconde a proposé une vue d'ensemble du dispositif. Si la première évaluation a conduit à une clarification $\mathrm{du}$ dispositif et à une nouvelle impulsion en faveur du secteur environnemental, en renforçant les exigences vis-à-vis de démarches plus spécifiquement tournées vers le secteur environnemental (notamment dans le domaine de la gestion des milieux), la seconde a beaucoup plus insisté sur les facteurs d'ambiguïté : ambiguïté de la démarche régionale vis-à-vis des politiques nationales en faveur de l'insertion, d'une part, et ambiguïté du comportement des structures d'accueil, y compris les collectivités locales, par rapport à l'utilisation du dispositif, de l'autre. De plus, l'enchevêtrement des financements et la concurrence des dispositifs nationaux, jugés plus attractifs pour les publics, ont finalement, malgré un bilan plutôt encourageant en termes d'emploi, conduit au choix de l'arrêt du dispositif.

16 La question de la concurrence entre les dispositifs nationaux et régionaux n'est pas négligeable, et a sans doute constitué un élément important dans le choix du recentrage. Le parallèle entre la politique nationale de l'emploi et la politique régionale est esquissé dans le tableau 1. Il montre assez clairement l'enchevêtrement des dispositifs successifs et la difficulté à distinguer clairement les actions menées par les différents échelons. 
Tableau 1 : Un parallèle entre les mesures de politique nationale de l'emploi et la politique régionale du Nord-Pas de Calais

\begin{tabular}{|c|c|}
\hline Politique de l'Etat & Politique de la Région \\
\hline $\begin{array}{l}1992 \text { - Plan } 900000 \text { CLD (Chômeurs Longue } \\
\text { Durée) } \\
\text { Création de postes d'insertion dans le secteur } \\
\text { de l'environnement. }\end{array}$ & \\
\hline $\begin{array}{l}1993 \text { - loi de juillet - " emplois verts ». } \\
\text { Objectif national : création de } 35000 \\
\text { emplois verts. } \\
\text { Dotation financière, au niveau des } \\
\text { DIREN (de } 1994 \text { à 1996) pour soutenir } \\
\text { les actions de protection de } \\
\text { l'environnement créatrices d'emplois. } \\
\text { Dans la Région NPC : les contrats verts } \\
\text { - } \\
\text { Programme reconduit jusqu'en 1996. } \\
\text { Au niveau régional (1994-1996) : } \\
\text {-134 projets, } \\
\text { - } 1342 \text { emplois (CES) }\end{array}$ & \\
\hline & $\begin{array}{l}1994 \text { : Création du dispositif « emploi } \\
\text { environnement». } \\
2 \text { objectifs : insertion et } \\
\text { environnement. } \\
\text { Résultat : } 40 \text { projets - } 500 \text { emplois } \\
\text { créés dont } 200 \text { stables (hors CES). } \\
\begin{array}{l}\text { Evaluation : difficultés d'atteindre les } \\
\text { objectifs au niveau social et environnemental. }\end{array}\end{array}$ \\
\hline
\end{tabular}




\begin{tabular}{|c|c|}
\hline & 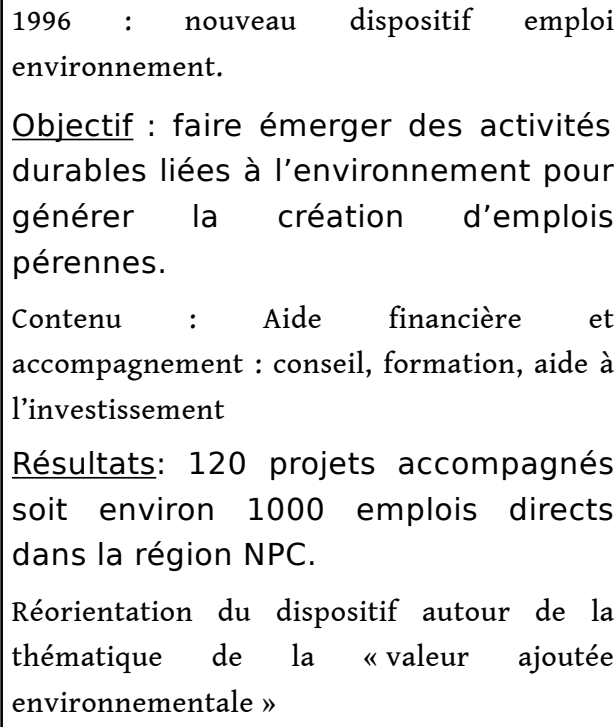 \\
\hline 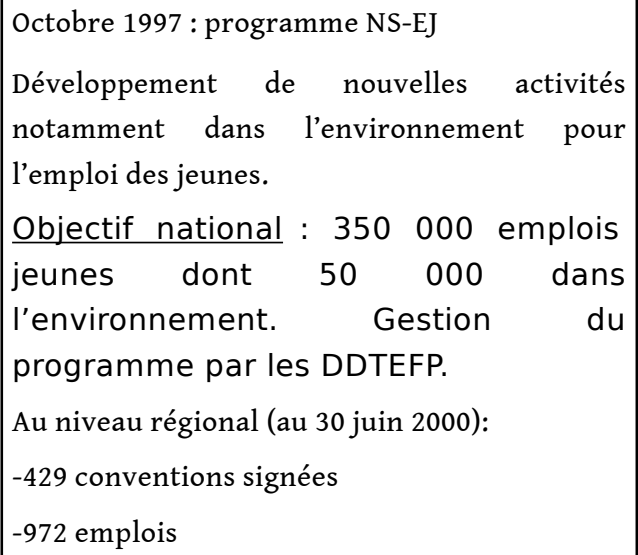 & \\
\hline & $\begin{array}{l}2002 \text { : Evaluation du dispositif conduisant à } \\
\text { son abandon. Recentrage vers le soutien } \\
\text { traditionnel à l'activité et le soutien aux } \\
\text { territoires (PLIE, PLDAIE, Agendas 21). }\end{array}$ \\
\hline
\end{tabular}

Source : d'après Falise et Robert (2001)

17 Comme on le voit dans le tableau 1, le parallèle entre les dispositifs nationaux et régionaux porte sur un seul domaine : l'emploi. L'environnement n'apparait pas comme un objectif spécifique. Cette remarque s'applique également à l'activité d'évaluation. $\mathrm{Si}$ les dispositifs ont bien été évalués par rapport à la question du nombre d'emplois créés et du statut de l'emploi et du secteur d'activité, aucune évaluation environnementale n'a été menée en propre, en dépit de la mise en place par la Région d'une réflexion explicite sur la question des indicateurs du développement durable ${ }^{3}$.

Pourtant, il semble bien que cette dimension ait été un enjeu important de la réussite du dispositif, du moins aux yeux des décideurs régionaux. Ceci est attesté par la présence, à plusieurs reprises, de remarques sur l'absence de culture environnementale des porteurs de projets dans les différents documents, notamment dans les deux documents d'évaluation. 
19 L'échec relatif du dispositif, le choix de son abandon, en dépit d'une volonté régionale très forte de promotion de l'emploi à l'échelon régional peuvent être expliqués par différentes "bonnes raisons» (Boudon, 2003) qui sont, aux yeux des acteurs, diverses: raisons financières (évaluation coût-avantage des mesures), difficultés de mise en œuvre spécifiques (incapacité à expliciter les attentes en matière de mise en œuvre). Ces raisons figurent clairement dans les documents d'évaluation des expériences. Néanmoins, un examen plus approfondi des ressorts de l'action collective permet sans doute d'aller plus loin dans la compréhension des logiques d'action développées autour de ce dispositif.

2. La construction d'une action collective territoriale

L'essai d'implantation d'une politique régionale emploi-environnement relève à l'évidence d'un problème d'action collective à l'échelle d'un territoire. Un des enjeux importants consiste, pour nous, à tenter d'en expliciter les ressorts. Une littérature très abondante et très complexe prend en charge la question de l'action collective, que ce soit du point de vue des individus, des institutions ou des inter-relations entre les deux (Commons, 1931, 1970 ; Coleman, 1990, Thévenot, 2006). Notre contribution ne vise pas à revenir sur l'analyse de l'action collective en général, même si nous tenons comme acquis les travaux en termes d'action publique située (Chatel et Salais, 2005), mais cherche à se positionner à l'interface de l'analyse des politiques publiques et de l'analyse territoriale. A ce titre, elle reconnaît, avec la théorie de la proximité, d'une part, le rôle fondamental des modes de coordinations (exprimés dans l'approche citée par les différentes formes de proximités) et d'autre part, l'importance de la construction de ces coordinations pour l'analyse territoriale (Pecqueur et Zimmermann, 2004 ; Colletis et Pecqueur, 2005).

21 L'analyse des modes de coordination, quoique nécessaire, reste toutefois insuffisante pour caractériser l'action publique locale. L'originalité de notre approche, par rapport à la vision sectorielle habituelle de la politique publique est de replacer l'action publique en regard des objectifs de développement économique territorial, puis, complémentairement, et à une échelle d'observation plus fine, de proposer une grille de lecture du comportement des acteurs insérés dans cet univers territorial. Si le territoire, pour reprendre l'analogie de Pecqueur et Ternaux (2006) joue bien un rôle de construit et de structuration du social, il convient, pour comprendre cette dynamique, de relier logiquement les comportements des acteurs et l'action territoriale de manière à identifier la (ou les) la logique(s) de construction de l'action collective territoriale.

L'analyse des systèmes productifs s'intéresse à la structuration économique du territoire par les entreprises et les réseaux de coordination qu'elles suscitent. Dans ce contexte, l'objectif des entreprises apparaît évident: il est déterminé prioritairement par des critères marchands. L'action des acteurs peut ainsi s'apparenter, au moins comme hypothèse de départ, à une logique marchande guidée par la recherche de l'intérêt privé. Dans le cas de la politique publique locale, les acteurs publics ne développent pas, bien entendu, une telle logique. On leur prête souvent, comme hypothèse a priori, une logique de défense d'une forme d'intérêt générique, appelé intérêt général ${ }^{4}$. Mais quel est-il ? Notre hypothèse est que pour les acteurs publics, l'intérêt général n'est ni une donnée exogène, ni un compromis d'intérêts particuliers. Il est lié au territoire. Les acteurs publics peuvent jouer un rôle de "production ", c'est-à-dire de transformation d'inputs (les ressources territoriales) en output (le développement territorial) ${ }^{5}$. Le moteur de cette transformation se situe dans la définition d'objectifs d'action collective. Il convient donc de mettre en évidence les liens entre comportement des acteurs (action publique) et modèles de développement territorial, tel que porté par ces acteurs. Un des problèmes est 
ainsi de spécifier les objectifs d'action collective autour duquel les agents économiques pourront se mobiliser ${ }^{6}$, et les mécanismes par lesquels ces objectifs peuvent induire (ou non) des formes de développement territorial.

Notre travail se concentre ici sur l'analyse des objectifs de l'action publique. Notre hypothèse est que cette politique emploi-environnement a constitué un élément de construction d'une démarche autonome et de structuration d'un objectif d'action collective pour la Région. L'étape de prise de conscience de deux objectifs antagonistes autour de cette politique emploi-environnement (celui de l'emploi pour le service public de l'emploi (SPE) et celui de l'environnement pour la Région) est un maillon essentiel qui permet de comprendre la formation de cette action publique et ses rapports au territoire. L'analyse de la genèse de cette opération passe par la compréhension de cette évolution. Comment se sont articulés ces deux objectifs ? Pourquoi ont-ils été considérés comme antagonistes, conduisant la Région à abandonner une politique qu'elle jugeait primordiale pour elle? L'enjeu de l'analyse consiste à comprendre le rôle des différents réseaux d'acteurs impliqués dans l'action publique, ainsi que l'articulation des actions des différents protagonistes issus des champs de la politique environnementale et de la politique d'emploi. Il s'agit de montrer qu'au final, l'échec de la politique menée peut s'interpréter comme une étape sans doute décisive, du processus de construction d'un objectif autonome de développement territorial.

Il faut bien se souvenir que la politique publique de l'emploi a un statut particulier, qui rend particulièrement complexe l'analyse de ses dimensions territoriales. Bien qu'elle soit toujours une compétence de l'Etat central, les actions sont de plus en plus territorialisées. Mais la territorialisation, si elle est simple déconcentration, ne signifie pas autonomie des choix territoriaux ${ }^{7}$. Parallèlement, la dimension territoriale peut être neutralisée si l'espace n'est pas considéré comme une dimension endogène des politiques publiques ${ }^{8}$ (Maillefert, 2003). La politique emploi-environnement se présente ainsi comme cas exemplaire de politique croisant les doubles dimensions du territoire (échelle locale d'action) et de l'initiative territoriale (échelle territoriale de définition des politiques). Pour replacer la réflexion dans le contexte de l'analyse des politiques publiques de l'emploi, nous souhaitons examiner, dans ce papier, certaines dimensions des relations entre acteurs, politique d'emploi et territoire. Ces dernières, qui témoignent d'une évolution plus générale des politiques publiques d'emploi en faveur de la subsidiarité sont analysées dans le tableau de l'annexe 1 (ligne 4 et 5 plus spécifiquement) (Salais, 1998; Millon-Delsol, 2002). Concernant le cas du Nord-Pas de Calais, deux axes seront privilégiés : la définition des objectifs, et la mise en œuvre de la politique.

2.1 Les objectifs de l'action collective

26 Comme il a été indiqué dans la première partie, les objectifs de la politique emploienvironnement ont évolué : entre 1993 et 1996, la Région a privilégié le développement d'un gisement d'emplois supposés exister dans le domaine environnemental. Puis les objectifs se sont déplacés vers "la création de valeur ajoutée environnementale", l'emploi devenant un levier d'action. Enfin, l'objectif d'emploi lui-même a disparu en tant que tel, après l'abandon du financement des programmes propres (comme les écogardes).

27 A l'origine, l'objectif de la politique régionale était de s'approprier un dispositif sectoriel pour en faire un dispositif territorial autonome et autosuffisant. Aux yeux des décideurs territoriaux, le financement d'une politique dans le secteur environnemental devait permettre la création d'emploi à l'échelle du territoire, l'emploi devant lui-même 
procéder d'une activité économique pérenne. C'est cette idée que les acteurs régionaux exprimaient par le terme de développement de «la valeur ajoutée environnementale». Les acteurs régionaux avaient simplement en tête l'idée de faire émerger une filière environnementale pérenne, autonome financièrement, et créatrice d'emploi ${ }^{9}$. Les aides apportées par la Région aux structures étaient donc conçues comme des dispositifs temporaires pour impulser une activité économiquement viable à (relativement court) terme.

Néanmoins, cet objectif dit de « création de valeur ajoutée environnementale » n'a jamais été ni clairement explicité, ni bien compris, et comme le souligne le premier rapport d'évaluation, il exprime une idée plutôt floue aux yeux des porteurs de projets, dotés d'une culture environnementale assez faible (qualifiée de "stade de la sensibilisation»). Ce flou résulte sans doute à la fois du caractère obscur de l'expression, mais peut-être aussi de la référence au domaine de l'environnement lui-même mal défini, oscillant entre une vision éco-centrée fondée sur l'environnement naturel et une vision anthropocentrée, privilégiant une entrée par la question des besoins humains ${ }^{10}$.

La lecture du programme initial régional de 1993, qui constitue le point de départ à la fois de l'approche régionale en matière d'action publique et la clé de lecture du dispositif (Zuindeau, 1997, p 63 et sq; Beca-environnement, 1997) fait apparaitre des éléments d'ambiguïté. Les priorités régionales sont clairement articulées autour d'une vision «naturaliste » qui réserve une place assez mineure aux questions liées à l'activité économique elle-même (tableau 2). On voit clairement que les mesures liées aux aspects économiques et plus particulièrement à l'emploi sont marginales et sont associées à une thématique assez vague et générale nommée « éducation, sensibilisation, formation ».

Tableau 2 : la politique régionale environnementale au début des années 1990

\begin{tabular}{|l|l|}
\hline $\begin{array}{l}\text { Mesures régionales } \\
\text { (7 mesures) }\end{array}$ & Déclinaison en actions (33 politiques) \\
\hline \hline Gestion de l'eau & $\begin{array}{l}\text { Connaissance des milieux } \\
\text { Réseaux qualité de l'air } \\
\text { Gestion globale de l'eau du ruissellement à la nappe } \\
\text { Programme d'assainissement du littoral } \\
\text { Contrats de rivière } \\
\text { Qualité des sols } \\
\text { Développement des pôles régionaux de compétence }\end{array}$ \\
\hline \hline $\begin{array}{l}\text { Reconquête et gestion } \\
\text { des espaces naturels }\end{array}$ & $\begin{array}{l}\text { Protection des paysages remarquables et requalification des espaces } \\
\text { et paysages dégradés } \\
\text { conservatoires } \\
\text { Poursuite et reconquête des friches industrielles } \\
\text { Action globale territorialisée dans le monde rural }\end{array}$ \\
\hline
\end{tabular}




\begin{tabular}{|c|c|}
\hline Ecologie urbaine & $\begin{array}{l}\text { Intervention sur l'aménagement et l'urbanisme } \\
\text { Espaces verts urbains et périurbains } \\
\text { Lutte contre les nuisances } \\
\text { Réduction des émissions polluantes } \\
\text { Soutien à l'instauration de comités de suivi sur tous les risques }\end{array}$ \\
\hline Technologies propres & $\begin{array}{l}\text { Sensibilisation développement et diffusion de la démarche audit- } \\
\text { environnement et système de management environnemental } \\
\text { Sensibilisation et développement des éco-produits et éco-labels } \\
\text { Favoriser l'intégration de l'environnement dans la conception et } \\
\text { l'aménagement des zones d'activités } \\
\text { Soutien à la diffusion et au transfert des innovations et des } \\
\text { technologies propres" } \\
\text { Développer une meilleure connaissance des pollutions industrielles } \\
\text {, dans le domaine de l'eau, de l'air, des déchets, etc }\end{array}$ \\
\hline Maîtrise de l'Energie & $\begin{array}{l}\text { Programme global de valorisation des énergies renouvelables } \\
\text { Programme d'efficacité énergétique dans les PME-PMI } \\
\text { Programme d'efficacité énergétique dans les Transports, } \\
\text { Programme d'efficacité énergétique dans l'Habitat Programme } \\
\text { d'efficacité énergétique dans le Tertiaire }\end{array}$ \\
\hline Maîtrise des déchets & $\begin{array}{l}\text { Mise en oeuvre du Schéma Régional de Valorisation des Déchets } \\
\text { Ménagers } \\
\text { Plan Régional de Maîtrise des Déchets Industriels } \\
\text { Plan Régional de Maîtrise des Déchets Hospitaliers et Médicaux } \\
\text { Plan Régional de Maîtrise des Déchets Agricoles et Agro- } \\
\text { alimentaires }\end{array}$ \\
\hline $\begin{array}{l}\text { Sensibilisation } \\
\text { Formation }\end{array}$ & $\begin{array}{l}\text { Création d'une Maison de l'Environnement } \\
\text { Création de Centres d'Initiation à l'Environnement } \\
\text { Développement des plans et chartes intercommunales } \\
\text { d'environnement. } \\
\text { Création d'un corps de gardes-nature et gardes-rivières } \\
\text { Soutien aux actions de sensibilisation, découverte et éducation des } \\
\text { associations naturalistes } \\
\text { Emploi-Environnement, formation insertion } \\
\text { Gestion et animation de la vie associative }\end{array}$ \\
\hline
\end{tabular}

Cette conception des relations entre environnement et économie se traduit logiquement par des attentes spécifiques de la part de l'acteur régional, qui ne sont pas partagées par les autres acteurs, particulièrement le Service Public de l'Emploi (SPE) et les associations d'insertion. On comprend alors aisément le sentiment d'insatisfaction des services régionaux vis-à-vis de l'activité proprement environnementale réalisée dans le cadre de la politique emploi-environnement, l'activité environnementale étant jugée peu 
conforme à la gestion écologique attendue. Ces divergences d'évaluation peuvent être traduites en deux types de conflit :

- conflit dans l'objectif de l'action publique : les acteurs de l'emploi ont comme objectif l'action d'intermédiation en matière d'emploi (ou plus précisément d'insertion), c'est-àdire le placement de personnes cibles. Or, une telle interprétation de la politique d'emploi a comme conséquence d'instrumentaliser le secteur environnemental en simple réceptacle de la politique, ou domaine d'accueil pour des publics cibles bénéficiaires de dispositifs d'emploi. L'aspect environnemental ou de " gestion écologique des milieux ", essentiel pour les décideurs régionaux, n'a qu'une importance mineure en regard des nécessités de placement des individus en insertion aux yeux des acteurs de l'emploi.

- conflit de norme de gestion: dans le cas (assez peu fréquent) où la question environnementale est jugée importante par les acteurs de l'insertion (cas des associations dédiées aux problèmes environnementaux de gestion des milieux), un autre problème apparait. Les collectivités locales, qui sont maitres-d'ouvrage refusent de payer le coût réel des services offerts par ces associations, qui sont majorés par rapport aux services liés à une gestion non écologique ou traditionnelle (la gestion écologique requiert plus de main-d'œuvre et peu de mécanisation). Cette attitude est confortée par le fait que bien souvent, les services ont d'abord été offerts à titre quasi-gratuit, du fait de l'existence de subventions. Pour pouvoir équilibrer leur gestion, les associations demandent, en contrepartie de leur action environnementale, à pouvoir bénéficier de financements pérennes (ce qui suppose l'acquisition de la reconnaissance du statut d'utilité publique pour les associations concernées), l'argument étant qu'elles relèveraient de l'intérêt général et donc des financements publics, au même titre que les transports publics ou l'éducation (Ernst et Young, 2002).

$33 \mathrm{Au}$ total, ces problèmes d'interprétation des objectifs témoignent d'un conflit sur la nature du modèle économique sous-jacent. Pour les uns (acteurs liés au SPE en particulier, mais aussi plus indirectement les associations environnementales), l'objectif principal est l'insertion, l'activité économique est associée au secteur non marchand et à des dispositifs d'insertion pérenne, dans lesquels ce sont les publics cibles qui tournent. Pour les autres (acteur régional en particulier), les activités doivent, dans un délai court, s'auto-financer, voire pouvoir intégrer le secteur marchand et correspondre à des emplois pérennes donc stables pour les individus concernés. Ce conflit de modèle économique se situe à un niveau assez général, mais traverse l'ensemble du dispositif. Il peut être précisé par une analyse plus fine du comportement des acteurs, mettant en perspective le rapport des individus au dispositif.

\subsection{Les registres de l'action collective}

La première partie a montré l'enchevêtrement des dispositifs successifs au cours des années $1990^{11}$, ce qui a occasionné un phénomène de concurrence, voire de cannibalisation entre les contrats verts (1994-1996), le dispositif «nouveaux servicesemplois jeunes » (1997-2002) et la politique régionale, dont les aides étaient généralement associées aux aides liées à ces contrats aidés. Malgré une politique de dégressivité des aides, il est clair que pour le budget de la Région, le coût ne pouvait se justifier qu'à condition de valoriser la double dimension spécifique du dispositif, à savoir la dimension environnementale et la pérennisation des emplois (qualité des emplois et durabilité des emplois) (Knockaert, Maillefert, 2004). 
pour ces deux dimensions, la Région a, de manière récurrente, formulé deux reproches aux bénéficiaires directs et indirects : un manque d'implication des structures associatives (plus intéressées par la dimension emploi) et un manque d'engagement de la part des collectivités territoriales (réticentes à accepter le surcoût généré par la gestion environnementale). S'ajoute à cela, la difficulté à assurer la pérennisation des emplois pris entre une logique "occupationnelle", un effet de substitution d'emplois (cannibalisme) ou encore une concurrence au secteur privé (Zuindeau, 1997).

Tous ces arguments et débats en termes de politique d'emploi sont bien connus et notre analyse de ce point de vue n'ajoute rien de nouveau. Par contre, le croisement avec la dimension environnementale permet de poser un regard différent sur les débats concernant ces politiques, qui concernent trois enjeux fondamentaux : la pérennisation des activités et des emplois, le rôle du secteur marchand, et la question de l'ancrage territorial ${ }^{12}$.

Depuis une vingtaine d'années, les expériences se multiplient dans le domaine de la politique territoriale et certains résultats paraissent convergents (OCDE, 2004) ${ }^{13}$. En particulier, la réussite des expérimentations semble essentiellement dépendre de la mobilisation des acteurs locaux, alors que la viabilité économique apparaît comme secondaire. Si on souhaite approfondir la question de la relation au territoire, il convient d'aller au-delà des ces résultats. Si, dans le cas qui nous intéresse, la question des financements n'apparaît pas comme essentielle, une dimension particulière nous semble fondamentale: elle concerne le contenu du registre d'action collective. Les registres d'action collective sont différents dans le cas des acteurs clés, la Région et les acteurs de la politique de l'emploi, comme le montre la lecture du tableau 3.

Tableau 3 : les registres d'action collective de la politique emploi-environnement. Le cas des acteurs régionaux et du SPE

\begin{tabular}{|c|c|c|}
\hline & Acteurs régionaux & $\begin{array}{l}\text { Acteurs du service public de } \\
\text { l'emploi }\end{array}$ \\
\hline Compétence & Action économique & $\begin{array}{l}\text { Politique d'emploi (insertion et } \\
\text { ciblage des publics) }\end{array}$ \\
\hline $\begin{array}{lll}\text { Nature } & \text { de } & \text { la } \\
\text { politique } & & \end{array}$ & Politique territoriale & Politique sectorielle \\
\hline Objectif & $\begin{array}{l}\text { Création d'activité (valeur ajoutée } \\
\text { environnementale) } \\
\text { Activité } \\
\text { économiquement viable }\end{array}$ & $\begin{array}{l}\text { Création d'emploi (insertion) } \\
\text { Activité durablement aidée }\end{array}$ \\
\hline $\begin{array}{l}\text { Vision des relations } \\
\text { entre } \\
\text { environnement et } \\
\text { emploi }\end{array}$ & $\begin{array}{l}\text { Environnement comme finalité, } \\
\text { Emploi comme moyen }\end{array}$ & $\begin{array}{l}\text { Emploi comme finalité, } \\
\text { environnement comme moyen }\end{array}$ \\
\hline
\end{tabular}

Développement durable et territoires, Varia (2004-2010) 


\begin{tabular}{|c|c|c|}
\hline $\begin{array}{l}\text { Logique d'action vis- } \\
\text { à-vis du public cible }\end{array}$ & $\begin{array}{l}\text { Logique de professionnalisation } \\
\text { (emploi durable comme emploi } \\
\text { pérenne et à terme, marchand) }\end{array}$ & $\begin{array}{l}\text { logique d'insertion (emploi } \\
\text { durable comme élément d'une } \\
\text { trajectoire d'insertion) }\end{array}$ \\
\hline $\begin{array}{l}\text { Logique d'évolution } \\
\text { du dispositif }\end{array}$ & $\begin{array}{l}\text { Développement d'une activité } \\
\text { environnementale marchande et des } \\
\text { emplois marchands pérennes dans } \\
\text { une logique de développement } \\
\text { durable. } \\
\text { Extension potentielle à toutes les } \\
\begin{array}{l}\text { activités économiques (logique } \\
\text { horizontale) }\end{array}\end{array}$ & $\begin{array}{l}\text { Développement d'une activité } \\
\text { d'insertion portée par des } \\
\text { activités non marchandes } \\
\text { durablement financées par des } \\
\text { fonds publics. } \\
\text { Extension liée aux publics } \\
\text { cibles (logique verticale) }\end{array}$ \\
\hline
\end{tabular}

Les acteurs proposent une lecture différente de chacun des critères. Néanmoins, cette différence se cristallise dans une dimension plus fondamentale, qui est celle de la vision $\mathrm{du}$ développement territorial, vision qui s'articule autour du critère concernant les logiques d'évolution du dispositif. Pour la Région, en particulier, la politique emploienvironnement devait être un moteur pour le développement de l'activité et servir d'exemple pour le développement territorial à venir. La réorientation de la politique a pris acte de l'échec de cette vision pour finalement proposer une démarche de soutien aux activités prenant en compte les questions environnementales (notamment le secteur des éco-industries).

Le changement d'orientation de l'action publique au cours de ces dix années d'expérimentation témoigne aussi certainement d'une évolution de la vision portée par les acteurs régionaux du développement économique et des relations entre économie et environnement, dont la politique emploi-environnement a été le vecteur. Clairement, la politique emploi-environnement constitue de ce point de vue une étape importante dans la construction, par les acteurs régionaux, d'une vision du développement territorial associant de manière spécifique la dimension environnementale et la dimension économique. Partis d'une vision très "naturaliste» (éco-centrée) des relations entre économie et environnement, les acteurs régionaux sont parvenus à une vision beaucoup plus centrée sur l'activité économique, et par là même développant un rapport très différent à l'espace naturel.

De ce point de vue, la politique emploi-environnement a participé à un processus de clarification des objectifs de développement dans lequel la vision du développement territorial, portée principalement par l'acteur régional, acteur clé de cette action collective, s'est construite et a évolué par confrontation avec une vision très différente, voire contradictoire, portée par les acteurs du SPE et les acteurs locaux de l'emploi. Un tel processus apparaît fondamental pour l'orientation des objectifs de développement du territoire, et à ce titre, il procède bien de la «révélation» du territoire (Colletis et Pecqueur, 2005), au sens où le territoire est "révélé en dynamique dans et par des situations de coordination situées dans le temps et dans l'espace » (Colletis et Pecqueur, art cit, $p$ 9). Cette interprétation est corroborée par une étude plus récente des politiques publiques en faveur de l'environnement, pour lesquelles une représentation convergente du développement territorial par l'acteur régional a pu être identifiée (Beaurain et Maillefert, dirs, 2006). 
41 L'analyse du processus d'action collective nous livre également un autre enseignement, d'ordre méthodologique cette fois, et qui relève plus du registre de la mise en œuvre (ou de ce que Salles et Colletis, 2007, nomment " niveau de la norme »). En effet, les registres d'action collective que nous avons proposés peuvent paraître triviaux, du moins en apparence, parce qu'implicitement connus des acteurs de terrain lors de la mise en œuvre du dispositif. Néanmoins, ils révèlent, eux-aussi, un enjeu de nature méthodologique. Si le dispositif avait fait l'objet d'une réflexion méthodologique explicite ex ante sur la nature de ces registres d'action, et ex post sur les résultats obtenus après la première année de fonctionnement, une méthodologie d'évaluation sur le double objectif d'emploi et de création de valeur ajoutée environnementale aurait pu être élaborée. Ainsi, certains ajustements auraient pu être réalisés de manière à mieux orienter les projets en fonction des attentes du dispositif. De cette façon, il est probable que le contenu du modèle économique aurait pu être clarifié, et avec lui, d'autres questions telles que la vision des relations entre économie et environnement, la dualité entre activités marchandes et non marchandes, l'objectif des politiques publiques, qui, restées latentes au cours du processus, n'ont été formulées qu'à l'occasion de l'arrêt du dispositif.

Conclusion

42 Le dispositif emploi-environnement, qui a été porté par la Région NPC pendant une dizaine d'années est un des dispositifs pour lesquels le croisement entre la dimension territoriale et la dimension d'action collective locale est particulièrement pertinente. Il s'agit à la fois de répondre à des objectifs de développement territorial et de recourir à l'action publique locale. Pour autant, le dispositif a évolué et son contenu initial s'est sensiblement affaibli, notamment du point de vue de la dimension emploi. Cette évolution peut être attribuée à l'ambiguïté concernant la définition de l'objectif, qui est apparu mal déterminé, à cause du flou entourant la manière de percevoir les rapports entre environnement et économie. Ce flou a suscité une interprétation différente des acteurs publics, qui ont, au final, repris le chemin des logiques sectorielles antérieurement appliquées, générant ainsi ce que Bartoli et Mériaux (2007) désignent comme un effet de «territorialisation concurrentielle ». Pour autant, le dispositif apparait de manière assez paradoxale, complètement cohérent avec les objectifs actuels de développement régional portés par la Région (modèle de la «reconquête environnementale»). Mais, comme le montre sans doute la lecture du dernier Schéma Régional d'Aménagement du Territoire (Conseil Régional du NPC, 2007), la perception des liens entre économie et environnement de la Région a elle-même beaucoup évolué.

Cette évolution de la perception du développement territorial par les acteurs régionaux nous invite à analyser la politique emploi-environnement avec un autre regard. Plutôt que d'analyser le dispositif comme un processus conduisant à l'échec d'une politique, nous l'avons interprété comme un processus de construction de ressources, dans lequel l'acteur régional, avec les autres acteurs de la politique publique a pu construire et faire évoluer sa vision du développement territorial, et ainsi procéder à l'opération de « révélation » du territoire, associée ici à une opération de définition d'objectifs collectifs de développement à l'échelle du territoire.

L'analyse a livré également un enseignement méthodologique à interpréter du point de vue du dispositif lui-même. Il aurait été certainement utile de déterminer de manière beaucoup plus explicite les attentes de la Région vis-à-vis du dispositif initial et de les formaliser dans une charte ou un guide méthodologique. En même temps, la construction d'une méthodologie transversale d'évaluation des différentes initiatives, qui a 
partiellement été réalisée lors de la première phase d'évolution du dispositif, aurait été certainement également très utile, pour les acteurs contribuant à la mise en place des mesures. Néanmoins, même si c'est aussi du côté de la méthodologie qu'il convient de chercher l'explication des difficultés de mise en œuvre, les hésitations méthodologiques traduisent également, et peut-être surtout, les étapes du processus de construction de ressources en jeu dans la mise en œuvre de l'action collective.

Annexe 1: Un essai de problématisation de l'action publique. De l'Etat Keynésien à l'Etat subsidiaire

\begin{tabular}{|c|c|c|c|c|}
\hline $\begin{array}{l}\text { Propriétés } \\
\text { de l'action } \\
\text { publique }\end{array}$ & Etat Keynésien & critiques & $\begin{array}{l}\text { Evolution des } \\
\text { pratiques de la } \\
\text { politique publique }\end{array}$ & $\begin{array}{l}\text { Pistes pour une } \\
\text { problématisation } \\
\text { nouvelle }\end{array}$ \\
\hline $\begin{array}{l}\text { Définition/ } \\
\text { réalisation } \\
\text { du bien } \\
\text { commun }\end{array}$ & $\begin{array}{l}\text { Plein-emploi } \\
\text { défini } \\
\text { statistiquement } \\
\text { (courbe de } \\
\text { Beveridge) }\end{array}$ & $\begin{array}{l}\text { Objectif } \\
\text { impossible à } \\
\text { atteindre } \\
\text { (montée du } \\
\text { chômage, CLD). } \\
\text { Le traitement } \\
\text { social du } \\
\text { chômage risque } \\
\text { de renforcer la } \\
\text { logique de } \\
\text { segmentation. } \\
\text { (Aucouturier, } \\
\text { 1998) }\end{array}$ & $\begin{array}{l}\text { Différenciation } \\
\text { des publics, } \\
\text { traitement en } \\
\text { amont de } \\
\text { l'exclusion. } \\
\text { Globalisation } \\
\text { (Mazel et alii, } \\
2000) .\end{array}$ & $\begin{array}{l}\text { Passage d'une } \\
\text { logique statique } \\
\text { (insertion) à une } \\
\text { logique } \\
\text { dynamique } \\
\text { (trajectoire). } \\
\text { Marchés } \\
\text { transitionnels } \\
\text { (Gazier, 1998). }\end{array}$ \\
\hline $\begin{array}{l}\text { Lien entre } \\
\text { action } \\
\text { individuelle } \\
\text { et le bien } \\
\text { commun }\end{array}$ & $\begin{array}{l}\text { Recherche } \\
\text { effective } \\
\text { d'emploi }\end{array}$ & $\begin{array}{lr}\text { Placement } & \text { des } \\
\text { chômeurs } & \text { dans } \\
\text { des dispositifs/ } \\
\text { contrainte } & \text { du } \\
\text { nombre } & \text { de } \\
\text { placements } & \text { pour } \\
\text { le SPE. }\end{array}$ & $\begin{array}{l}\text { Objectifs de baisse } \\
\text { des chiffres de } \\
\text { catégories } \\
\text { spécifiques de } \\
\text { chômeurs. }\end{array}$ & \begin{tabular}{lr}
\multicolumn{2}{l}{ Logique de guichet } \\
/ de projet. \\
Nécessité & de \\
construction & de \\
parcours & \\
d'insertion & \\
(activation & des \\
capacités). &
\end{tabular} \\
\hline $\begin{array}{l}\text { Principe de } \\
\text { justification } \\
\text { de l'action } \\
\text { publique }\end{array}$ & $\begin{array}{l}\text { Chômage } \\
\text { involontaire } \\
\text { Principe de } \\
\text { Redistribution }\end{array}$ & $\begin{array}{ll}\text { Inéquité des } \\
\text { dispositifs } \\
\text { (sélection des } \\
\text { demandeurs, } \\
\text { formations- } \\
\text { parking). }\end{array}$ & $\begin{array}{l}\text { Affinement des } \\
\text { mesures. } \\
\text { Territorialisation } \\
\text { et autonomisation } \\
\text { de l'action } \\
\text { publique locale. }\end{array}$ & $\begin{array}{l}\text { Approche } \\
\text { individualisée, } \\
\text { transversale et } \\
\text { territorialisée } \\
\text { (Farvaque, 2002). } \\
\text { enjeu : définition } \\
\text { d'une dimension } \\
\text { territoriale } \\
\text { autonome. } \\
\text { Expérimentation. }\end{array}$ \\
\hline
\end{tabular}




\begin{tabular}{|c|c|c|c|c|}
\hline $\begin{array}{l}\text { Logique } \\
\text { d'action } \\
\text { individuelle }\end{array}$ & Rationalité & $\begin{array}{l}\text { Dérive vers le } \\
\text { calcul } \\
\text { économique pur } \\
\text { ou la logique de la } \\
\text { compassion } \\
\text { (intérêt extérieur } \\
\text { ex la question de } \\
\text { l'exclusion). }\end{array}$ & $\begin{array}{l}\text { Raisonnabilité } \\
\text { (comprendre les } \\
\text { logiques d'usage } \\
\text { et } \\
\text { d'interprétation } \\
\text { d'une mesure). }\end{array}$ & $\begin{array}{l}\text { Logique de } \\
\text { l'engagement } \\
\text { (Sen, 1993) } \\
\text { Enjeu: définition } \\
\text { der critères } \\
\text { d'efficacité. }\end{array}$ \\
\hline $\begin{array}{l}\text { Norme de } \\
\text { l'Etat } \\
\text { (Relation } \\
\text { entre } \\
\text { l'action } \\
\text { collective et } \\
\text { la formation } \\
\text { du bien } \\
\text { commun) }\end{array}$ & $\begin{array}{l}\text { Etat extérieur } \\
\text { (bien commun } \\
\text { déterminé en } \\
\text { dehors de } \\
\text { l'action } \\
\text { collective cf Etat } \\
\text { comme dictateur } \\
\text { bienveillant). }\end{array}$ & $\begin{array}{l}\text { Inefficacité de } \\
\text { l'Etat. Dérive } \\
\text { bureaucratique. }\end{array}$ & $\begin{array}{l}\text { Construction } \\
\text { collective du bien } \\
\text { commun. }\end{array}$ & $\begin{array}{l}\text { Jugement } \\
\text { posteriori. } \\
\text { Développement } \\
\text { des niveaux } \\
\text { intermédiaires } \\
\text { (territoires, } \\
\text { groupes } \\
\text { intermédiaires). } \\
\text { Etat subsidiaire } \\
\text { (Millon-Delsol, } \\
\text { 1992). }\end{array}$ \\
\hline
\end{tabular}

\section{BIBLIOGRAPHIE}

Aucouturier A.L (1998), «Evaluation des politiques d'emploi et action publique. L'exemple de l'aide aux chômeurs créateurs d'entreprise ». Thèse pour le doctorat, université Paris X-Nanterre, $509 \mathrm{p}$.

Beaurain Ch., Maillefert M. (dirs) (2006), « Politiques régionales de l'environnement et développement économique, une approche territoriale », Rapport pour le MEDD, juin, 187 p. BECA-environnement (1997), l'évaluation du Dispositif régional emploi-environnement 1994-1995, Rapport pour le Conseil Régional de NPC.

Bartoli J.R, Meriaux O, (2007), « Les politiques de l'emploi au risque de la territorialisation concurrentielle », in FAURE A; Negrier E (DIRS), Les politiques publiques à l'épreuve de l'action locale. Critiques de la territorialisation, L'Harmattan, $p p$ 35-42.

Berthet T, Cuntigh P, Guitton c. (2002), La politique d'emploi au prisme des territoires, DARES, document d'études $n^{\circ} 59$.

Boulangé Ch., Mairesse C. (2003), Le développement durable et l'emploi-environnement dans le Nord-Pas de Calais, Master GPE, Université Lille 3.

Boudon R. (2003), Raisons. Bonnes raisons, PARIS, PUF. 
Chatel E, SALAIS R « Introduction », IN Chatel E, Kirat TH, Salais R (DIRS). (2005), L'action publique et ses dispositifs, L'Harmattan, PP 11-19.

Coleman J. (1990), Foundations of Social Theory, Havard university Press.

Colletis G. ET Pecqueur B. (2005), « Révélation de ressources spécifiques et coordination située », Économie et Institutions, $\mathrm{n}^{\circ} 6-7,1^{\mathrm{er}}$ et $2^{\text {nd }}$ semestres.

Commons J.R (1931), Institutionnal Economics, American Economic Review, 21, P. 648-657.

Commons J.R, 1970 [1950], The Economics of Collective Action, The University of Wisconsin Press.

Conseil économique et social (2004), La " judiciarisation de l'économie », rapport présenté par J.-P Noury.

Conseil européen de Lisbonne (2000), Synthèse, mars, www. Europa.eu.int

Conseil régional du NPC (1994), Dispositif régional emploi-environnement.

Conseil régional du NPC (1996), Dispositif régional emploi-environnement.

Conseil régional du NPC (1998), L'emploi et l'environnement.

Conseil régional du NPC (1999), Mise en œuvre de la nouvelle politique économique.

Conseil régional du NPC (2000), Orientations stratégiques en matière d'environnement.

Conseil régional du NPC (2006), Budget Primitif pour 2006.

Conseil régional du NPC (2007), Schéma Régional d'Aménagement et de Développement du Territoire, Objectif 2020, adopté le 22 nov 2006.

Constantinesco V (1991), Le principe de subsidiarité : un passage obligé vers l'Union européenne? Mélanges Boulouis.

Ernst et Young (2002), Evaluation du dispositif régional emploi-environnement, rapport pour le conseil régional du NPC.

Falise V., Robert V. (2001), Politiques publiques d'emploi et environnement. Le cas de la RÉGION NPC. Master GPE, Université Lille 3.

Farvaque N. (2002), « Le déploiement local de l'action publique en faveur de l'insertion des jeunes : le rôle des dipositifs. Le cas d'une mission locale ", Communication aux deuxièmes rencontres inter-universitaires de l'économie sociale et solidaire, Lyon, 5-7 février.

Feral P.A. (1996), le principe de subsidiarité dans l'Union européenne, RD publ.

Hascoet G. (1997), Territoires, emploi et développement durable, Rapport parlementaire.

Knockaert S., Maillefert M. (2002), « L'emploi durable, un concept pertinent à l'échelon territorial ? ", Cahiers Lillois d'économie et de sociologie, n³7, « Développement Durable et territoire », pp 133-150.

Knockaert S., Maillefert M. (2004) « Qu'est-ce que l'emploi durable? », Natures, Sciences, Sociétés, 12, 2, pp 135-145.

Laroque G, Salanié B (2000), « une décomposition du non emploi en France », Economie et statistique, $\mathrm{n}^{\circ} 331$, pp 47-66. http://www.insee.fr/fr/ffc/docs_ffc/ES331C.pdf.

Maillefert M. (2002a) «Etat, action publique et territoire : quels enjeux pour la politique d'emploi territoriale? ", Sciences de la société, $\mathrm{n}^{\circ} 57$, « autour du développement durable », 2002, pp 77-94. 
MAILLEFERT M (2002b), Représentations et action : de la construction du collectif à la politique publique. Note pour l'obtention de l'HDR, Université Paris X.

Maillefert M. (2003) «La territorialisation de la politique d'emploi en France », Allemagne d'Aujourd'hui, $n^{\circ} 163$, dossier spécial « les politiques publiques de l'emploi en Allemagne, France et en Pologne", janvier-mars 2003, pp 62-78.

Millon-Delsol C. (1992), L'état subsidiaire, Paris, PUF., 227 p.

O.C.D.E. (2004), Environment and employment, an assessment. Publications de L'O.C.D.E

Pecqueur B., Ternaux P. (2006), « Des ressources territoriales aux interactions entre acteurs ", cinquièmes journées de la proximité, Bordeaux, 28-30 juin.

Pecqueur B. et Zimmermann J.-B. éds., (2004), Économie de proximités, Hermes Science Publications, Lavoisier, Paris.

Regards économiques sur le droit (2005), Problèmes économiques, $n^{\circ} .2872,30$ mars, la documentation Française.

Salais R. (1998), « A la recherche du fondement conventionnel des institutions », in. SALAIS R., Chatel E., Rivaud-Danset D., in Institutions et Conventions. La réflexivité de l'action économique, Paris, Ed. de l'EHESS, pp 255-291.

Salles M. Colletis G. (2007), « Représentations de l'entreprise dans les systèmes d'information statistique et décision dans les collectivités territoriales », Droit et Société, n65, pp 53-74.

SEN A. (1993), Ethique et économie, Paris, Puf, 360 p.

Théret B. (2003), « Institutionnalismes et structuralismes: oppositions, substitutions ou affinités électives ? ", Cahiers d'économie politique, nº spécial, décembre, pp.1-33.

Thévenot L. (2006), L'action au pluriel, sociologie des régimes d'engagement, Paris, La découverte.

Zuindeau B. (1997), Recherche-action portant sur la promotion des gisements d'emplois en environnement , Rapport pour le Conseil régional du NPC.

\section{ANNEXES}

Annexe 1: Un essai de problématisation de l'action publique. De l'Etat Keynésien à l'Etat subsidiaire

\section{NOTES}

1.G. Laroque et B. Salanié (2000) sur ravaux antérieurs de modélisation entaliste (et du modèle néoe réel du phénomène , comme dans l'économétrie l'appui sur des catégories très connotées par les résultats.

2. $\mathrm{n}$ but de développement de t été répartis par les DIREN entre 
PE a montré que $75 \%$ de ces emplois se

és ou contrats de retour à

l'emploi.

3. $n$ compte du développement

re identifié. Il a servi sans doute à

e connaissance, le programme

ype de critères, permettant de

valoriser les résultats en terme de développement durable.

4. Notre propos n'est pas de discuter des formes que prend cet intérêt au regard des intérêts privés, mais il convient évidemment de garder à l'esprit les critiques de l'action publique « surplombante » ou, à l'inverse de l'action publique intéressée qui, chacune à leur manière critiquent l'action publique telle que l'analyse économique l'a appréhendée jusque dans les années quatre-vingt.

5. Pour parler le langage de la proximité, on dira que l'action publique se présente comme un processus (potentiel) d'activation de ressources à l'échelle d'un territoire (Colletis et Pecqueur, 2005). Ce processus est impulsé un acteur-clé, ici, l'acteur Régional. Ce dernier en effet impulse (en partie) la politique, l'anime, la (co)finance et négocie les différents éléments de mise en œuvre avec d'autres agents : l'Etat pour les financements (SPE), les partenaires locaux (associations par exemple, voire cabinets de consultants pour l'appui au montage des dossiers) pour la mise en oeuvre, etc.

6. Le dernier SRADT de la Région Nord-Pas de Calais utilise explicitement le terme « d'intérêt régional » (SRADT, 2007).

7. On distingue traditionnellement la territorialisation de politiques (dimension global/ local) de la politique de territoire (dimension spatiale) (C'est la dimension verticale des dispositifs.

8. Les dispositifs qui jouent sur le coût du travail (allègement des charges par exemple) se réfèrent à la dimension marchande, concurrentielle et a spatiale de la ressource en travail. Le croisement de la dimension spatiale (espace abstrait, concret) et de la nature de la politique d'emploi (globale, locale) permet de définir quatre cadrans dans lesquels peuvent s'inscrire différents dispositifs de la politique d'emploi (Maillefert, 2003).

9. uation pour la Région en 2002, est

ue l'action

insuffisamment exploitée du fait de

uilibre fragile des activités susceptibles

d'être générées » (Ernst et Young, 2002, p 9).

10. C'est la vision du rapport Brundtland.

11. e à la distinction de L. Thévenot (2006,

$\mathrm{s}:$ La catégorie du registre se

ime. Elle nous semble adéquate

dire des représentations de

ntéresse à l'individu et ses

conduites).

12. Larché ou l'arrêt des structures, ce

d'insertion. Zuindeau (1997)

rs professionnels et

développement durable, et dans ce

urabilité des emplois apparaît pertinent.

Développement durable et territoires , Varia (2004-2010) 
es pour qualifier la dimension de

ement territorial, et le partenariat

(Knockaert, Maillefert, 2002).

13.es, dont 169 en Europe et 22

réussite. Les conditions

enantes, implication des

d'une coalition d'intérêts,

ction et agenda commun,

nnement. Les conditions de

isation des parties prenantes, le

rofessionnel et la

communication.

\section{RÉSUMÉS}

Cette contribution examine un dispositif de politique publique particulier mis en place par la Région Nord-Pas de Calais entre 1993 et 2002. Ce dispositif, appelé politique emploienvironnement, croise la dimension territorialisée des politiques publiques et la dimension d'action collective locale. L'examen des logiques d'action collective portées par les différents acteurs montre que la principale difficulté de mise en œuvre a reposé non pas sur les objectifs mêmes du programme, cohérents avec ceux de la Région, mais plutôt sur les registres d'action collective développés par les divers acteurs impliqués dans le dispositif, chaque acteur développant sa propre interprétation des objectifs, rendant le projet par là-même paradoxal dans sa mise en oeuvre. En même temps, et malgré son échec, ce dispositif a participé à la construction de la politique de développement territorial de la Région, contribuant par là même à un processus de « révélation » du territoire.

This paper examines a special public policy plan put into place in the Nord-Pas de Calais Region between 1993 and 2002. This plan called Employment-Environment Policy mixed the territorial and the local collective action dimensions. Examination of the ethos for collective action put forth by the various participants shows that the main difficulty for implementation arose, not from the very objectives of the programme that were in harmony with the Region's ones, but rather from the kinds of collective actions developed by the various players involved in the plan.

\section{INDEX}

Keywords : sustainable development, collective action, employment policy

Mots-clés : développement durable, action collective territoriale, politique d'emploi 


\section{AUTEURS}

\section{MURIEL MAILLEFERT}

Muriel Maillefert est Maître de conférences, Lille 3 et Clersé-Meshs, UMR 8019 ;

muriel.maillefert@univ-lille3.fr

\section{NICOLA SCRENCI}

Nicola Screnci est Juriste et consultant en développement durable 